\title{
Asymptotic Solutions of Differential Equations with Transition Points or Singularities*
}

\author{
A. ERdelyi \\ Department of Mathematics, California Institute of Technology, Pasadena, California
}

(Received December 7, 1959)

\begin{abstract}
Asymptotic, solutions of $d^{2} y / d x^{2}+\left[\lambda^{2} p(x)+r(x, \lambda)\right] y=0$ are found when $\lambda$ is a large parameter and $r$ is "small" in comparison with $\lambda^{2} p$, except at a single point where either $p$ has a simple zero, or $p$ a pole of the first order and $r$ a pole of the second order. The results are applied to Bessel functions, and to Hermite and Laguerre polynomials. The resulting asymptotic forms are valid uniformly in $x$.
\end{abstract}

\section{INTRODUCTION}

$\mathbf{I}^{\mathrm{N}}$ $\mathrm{N}$ many problems of applied mathematics one needs approximations to solutions of the differential equation,

$$
d^{2} y /\left(d x^{2}\right)+\left[\lambda^{2} p(x)+r(x, \lambda)\right] y=0
$$

where $\lambda$ is a large parameter, and $r(x, \lambda)$ is "small" in comparison with $\lambda^{2} p(x)$. In this paper, $x$ will be a real variable ranging over a finite or infinite open interval $(a, b)$.

The earliest investigation of such an approximation is due to Liouville (1837) who encountered (1.1) in his work on what is now called the Sturm-Liouville problem. In Liouville's case, $\lambda^{2} p(x)>0$ throughout $(a, b)$, and the solutions are shown to be oscillatory. Liouville's method can also be applied when $\lambda^{2} p(x)<0$ throughout $(a, b)$ and it turns out that in this case the solutions show a monotonic behavior in the sense that no nontrivial solution can vanish more than once. A brief description of Liouville's method will be found in [3, Chap. IV]; here it will be sufficient to note that that method is based on a comparison of (1.1) with a differential equation with constant coefficients, and that the technique of the comparison involves a change of variables followed by a conversion of the differential equation into an integral equation of Volterra type.

The behavior of the solutions of (1.1) is much more involved if $\lambda^{2} p(x)$ changes its sign in $(a, b)$, say at a point $c$. This point separates an interval in which the solutions of (1.1) are monotonic from one in which they are oscillatory; and the transition from the one kind of behavior to the other takes place in the immediate vicinity of $c$. Such a change of sign commonly takes place if $c$ is a zero of $p(x)$, in which case it is called a transition point or a turning point of the differential equation, or if $c$ is a singularity of $p(x)$, and hence of the differential equation.

$\mathrm{H}$. Jeffreys encountered a turning point problem in 1923 when he investigated oscillations of water in an elliptic lake, and he developed approximations for that case [10, Sec. 17.131]. A few years later, turning point problems arose in connection with the one-dimensional

* This paper is based on several reports by the author and others. These reports were prepared under contract with the Office of Naval Research and are listed with all other references in the Bibliography at the end of this paper under $1,3,4,5,8,9$, 19,20 . References appear in the text in brackets.
Schrödinger equation, and the $W K B$ method was invented to solve them. Liouville's method was adapted to differential equations with transition points by $\mathrm{R}$. E. Langer. Among the many investigations carried out in this field those of Langer, T. M. Cherry, and F. W. J. Olver are especially pertinent to our purpose. A brief review of the relevant literature up to 1951 is given in [1], and general descriptions of the comparison method, in [5] and [14].

More recently, singularities of (1.1) at which $\lambda^{2} p(x)$ changes its sign have also been studied, the principal contributions being due to E. D. Cashwell, N. D. Kazarinoff, and R. McKelvey, Olver, and C. A. Swanson.

In this paper we shall assume that $\lambda$ is a large positive parameter, $(a, b)$ a finite or infinite open interval, and $p(x)$ is real and changes its sign at $c, a<c<b$. For the sake of definiteness, we mostly take $p(x)<0$ for $a<x<c$, and $p(x)>0$ for $c<x<b$. At $c$ itself, $p(x)$ has either a simple zero or a simple "pole." Comparison with a differential equation with constant coefficients does not lead to adequate approximations in either of these two cases, and it becomes necessary to use comparison equations which themselves have a transition point or a singularity, as the case may be.

The simplest comparison equation with a transition point is

$$
d^{2} w / d t^{2}+\lambda^{2} t w=0
$$

which is closely related to Airy's equation. Solutions of (1.2) are

$$
A i\left(-\lambda^{i} \omega^{m} t\right), \quad B i\left(-\lambda^{i} \omega^{m} t\right),
$$

where $A i$ and $B i$ are the Airy functions [15], $m$ is an integer, and $\omega=e^{2 \pi i / 3}$.

The simplest comparison equation with a singularity would seem to be the differential equation,

$$
d^{2} w / d t^{2}+\lambda^{2} t^{-1} w=0,
$$

which is closely related to Bessel functions of order unity. However, it turns out that the adoption of this comparison equation necessitates undue restrictions on $r(x, \lambda)$. For this reason, we shall use a somewhat more elaborate comparison equation,

$$
\frac{d^{2} w}{d t^{2}}+\left(\frac{\lambda^{2}}{t}+\frac{1-\nu^{2}}{4 t^{2}}\right) w=0,
$$


in which $\nu$ is a nonnegative constant to be chosen later. (1.4) is also closely related to Bessel's differential equation, and each of the functions,

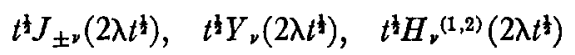

in the usual notation of Bessel functions, is a solution of (1.4).

In this paper $\lambda, x$ and $p(x)$ are assumed to be real. It may be noted that results for complex values of $\lambda$ are available in the reports on which this paper is based and results for complex $x$ and $p(x)$ in some of the references given at the end of the paper.

\section{TRANSITION POINTS}

\section{Outline of the Method and Results}

We introduce a new variable $x$ in (1.2) by the substitution,

$$
t=\phi(x), \quad w=\left[\phi^{\prime}(x)\right]^{3} Y,
$$

thus changing (1.2) in

where

$$
\left(d^{2} Y / d x^{2}\right)+\left(\lambda^{2} \phi \phi^{\prime 2}+\frac{1}{2}\{\phi, x\}\right) Y=0,
$$

$$
\{\phi, x\}=\left(\phi^{\prime \prime \prime} / \phi^{\prime}\right)-\frac{3}{2}\left(\phi^{\prime \prime} / \phi^{\prime}\right)^{2}
$$

is the Schwarzian derivative of $\phi$ with respect to $x$. The differential equations (1.1) and (2.1) will be nearly identical for large $\lambda$ if we choose $\phi$ so that

$$
\phi \phi^{\prime 2}=p(x) .
$$

With this choice of $\phi,(1.1)$ may be written in the form,

where

$$
\left(d^{2} y / d x^{2}\right)+\left(\lambda^{2} \phi \phi^{\prime 2}+\frac{1}{2}\{\phi, x\}\right) y=F(x, \lambda) y,
$$

$$
F(x, \lambda)=\frac{1}{2}\{\phi, x\}-r(x, \lambda) .
$$

In this exposition we shall discuss (2.4) rather than (1.1) and will express conditions of validity with reference to $\phi$ and $F$ rather than $p$ and $r$. Once $\phi$ has been determined from (2.3), $F$ is also known and it is usually easy to verify that the conditions of validity are satisfied.

If $F(x, \lambda)$ is small in comparison with $\lambda^{2} \phi \phi^{2}+\frac{1}{2}\{\phi, x\}$, then (2.1) and (2.4) will be nearly identical, and we shall expect the known solutions of (2.1) to be good approximations to solutions of 2.4. In order to estimate the difference $y-Y$, we regard for the moment the right hand side of (2.4) as a given function of $x$, and apply the method of variation of parameters to (2.4), obtaining

$$
y(x)=Y(x)+\int_{x^{\prime}}^{x} K(x, t) F(t, \lambda) y(t) d t,
$$

where $x^{\prime}$ is some fixed point of the interval, $Y(x)$ is a solution of (2.1), and $K(x, t)$ is the Green's function of (2.1). For a fixed $t$ in $(a, b), K(x, t)$, as a function of $x$, satisfies (2.1) together with the initial conditions $K(t, t)=0,(\partial K / \partial x)(t, t)=1$.
In order to find approximations for a solution of (2.4), which is characterized by initial conditions at an interior point of $(a, b)$, or by its behavior as $x \rightarrow a$ or $x \rightarrow b$, we choose $x^{\prime}$ to be the point in question and $Y(x)$ to be that solution of (2.1) which is characterized by the same conditions as $y(x)$. We then establish the existence of a solution of the integral equation (2.6), prove that this solution is differentiable and satisfies (2.4), obtain a rough estimate of $y(x)$, and use this estimate in the integral in (2.6) to obtain an improved estimate of $y(x)-Y(x)$. In order to carry out this program, it will be necessary to introduce some notations.

Clearly, $\phi$ must be assumed to be three times differentiable on $(a, b)$, and it will also be assumed that $\phi^{\prime}$ is either positive throughout $(a, b)$, or else negative throughout $(a, b)$. We set

$$
\begin{aligned}
\omega & =e^{2 \pi i / 3} \\
\Phi & =\Phi(x)=\Phi(x, \lambda)=\left|\phi^{\prime}(x)\right|^{3}\left(1+\lambda^{1 / 6}|\phi(x)|^{1}\right), \\
\theta_{m} & =\theta_{m}(x)=\theta_{m}(x, \lambda)=-\lambda^{\frac{3}{3} \omega^{m}} \phi(x), \quad m=0, \pm 1 \\
\theta_{2} & =\theta_{0}=\theta \\
\sigma & =\frac{2}{3} \theta^{\frac{3}{3}}, \quad \sigma_{m}=\frac{2}{3} \theta_{m^{\frac{3}{2}}}, \quad m=0, \pm 1,2 .
\end{aligned}
$$

It is seen from (1.3) that

$$
\begin{aligned}
Y_{m}(x) & =\left|\phi^{\prime}\right|^{-\frac{1}{k}} A i\left(\theta_{m}\right) \quad m=0, \pm 1 \\
Y_{2}(x) & =\left|\phi^{\prime}\right|^{-\frac{1}{1}} B i(\theta)
\end{aligned}
$$

are solutions of (2.1). The approximations to $y$ will be expressed in terms of these solutions. In the error estimates certain complications arise due to the zeros of the Airy functions. In order to cope with these, we set

$$
\begin{aligned}
& \widetilde{Y}_{m}(x)=\begin{array}{l}
Y_{m}(x) \quad \text { except when } \theta_{m}<0 \\
\left|\phi^{\prime}\right|^{-1}\left[\left|A i\left(\theta_{m}\right)\right|^{2}+\left|B i\left(\theta_{m}\right)\right|^{2}\right]^{\frac{1}{2}} \text { if } \theta_{m}<0
\end{array} \\
& \frac{d}{d x}\left[\left|\phi^{\prime}\right|^{\prime} Y_{m}(x)\right] \quad \text { except when } \theta_{m}<0 \\
& \tilde{Z}_{m}(x)= \\
& \lambda^{\prime} \phi^{\prime}\left[\left|A i^{\prime}\left(\theta_{m}\right)\right|^{2}+\left|B i^{\prime}\left(\theta_{m}\right)\right|^{2}\right]^{z} \text { if } \theta_{m}<0 \text {. }
\end{aligned}
$$

These functions will be used in estimates only. $\widetilde{Y}_{m}$ is not a solution of $(2.1)$, and $\widetilde{Z}_{m}(x)$ is not the derivative of $\left|\phi^{\prime}\right|^{\frac{1}{3}} Y_{m}$, if $\theta_{m}<0$.

Lastly, we set

$$
G(x, \lambda)=\lambda^{3} F(x, \lambda)[\Phi(x, \lambda)]^{-2} .
$$

$x$ and $t$ range over $a, b$; and $\lambda>0$. All bounds, including those implied by the $O$ symbols, are independent of $x, t$, $\lambda . C$ is a generic bound which may be different at each occurrence.

It is seen from (2.3) that $\phi$ and $p$ vanish at the same point, and have the same sign where they do not vanish. Thus $\phi^{\prime}(x)>0$ if $p(x)<0$ for $a<x<c$ and $p(x)>0$ for $c<x<b$, and $\phi^{\prime}(x)<0$ if $p(x)>0$ to the left, and $p(x)<0$ 
to the right of $c$. For the former case, we shall establish the following result:

If (i) $\phi$ is three times continuously differentiable, and $\phi^{\prime}(x)>0$, for $a<x<b$, and $\phi(c)=0$; (ii) for each fixed positive $\lambda, F(x, \lambda)$ is a continuous function of $x$ for $a<x<b$; (iii) $|G(x, \lambda)| \leq \Lambda(\lambda) g(x)$ where $(1+\lambda)^{-1} \Lambda(\lambda)$ is $a$ bounded function of $\lambda$ for $\lambda>0$, and $g(x)$ is integrable over $(a, b)$; and if we set

$$
x_{0}=a, \quad x_{ \pm 1}=b, \quad x_{2}=c,
$$

then the integral equation,

$$
y_{m}(x)=Y_{m}(x)+\int_{x_{m}}^{x} K(x, t) F(t, \lambda) y(t) d t
$$

has a unique solution for each $m=0, \pm 1,2$ and each $\lambda>0$, this solution is trerice continuously differentiable and satisfies (2.4) for $a<x<b$, and

$$
y_{m}(x)=Y_{m}(x)+O\left[\frac{\Lambda(\lambda)}{\lambda} Y_{m}(x) \int_{x_{m}}^{x} g(t) d t\right]
$$

for $\lambda>0$ and $a<x<b$.

This result can easily be adapted to the case when $p(x)>0$ for $a<x<c$ and $p(x)<0$ for $c<x<b$.

If $\phi^{\prime}(x)<0$,

$$
x_{0}=b, \quad x_{ \pm 1}=a, \quad x_{2}=c
$$

and otherwise the conditions (i) to (iii) hold, then the result enunciated in the foregoing also holds.

It follows from (2.15) that $Y_{m}(x)$ is an asymptotic representation of $y_{m}(x)$ as $\lambda \rightarrow \infty$, which holds uniformly in $x$; and also for fixed $\lambda$ as $x \rightarrow x_{m}$. This is an especially valuable feature when solutions of (2.1) are characterized in terms of their behavior as $x \rightarrow x^{\prime}$. It can further be proved that $y_{m}{ }^{\prime}$ is also represented asymptotically by $Y_{m}^{\prime}[4]$.

$Y_{m}$ may be regarded as a first asymptotic approximation to $y_{m}$. Higher approximations may be obtained either by expanding the solution in descending powers of $\lambda$ (Langer $[13]$, Olver $[17,18]$ ) or else by using in place of $\phi$ a change of variable which itself depends on $\lambda$ (Cherry [2]).

\section{Auxiliary Estimates}

In the derivation of (2.15) certain estimates are needed. These are in essence estimates on Airy functions. The reader is referred in this connection to [15] and $[16]$.

$A i(z)$ and $B i(z)$ are entire functions. All zeros of $A i(z)$ and $A i^{\prime}(z)$ are situated on the negative real axis and all zeros of $B i(z)$ or $B i^{\prime}(z)$, either on the negative real axis or in one of the two sectors $\pi / 3< \pm \arg z<\pi / 2$. Moreover, on the negative real axis, the zeros of $A i(z)$ and $B i(z)$ interlace, and so do the zeros of $A i^{\prime}(z)$ and $B i^{\prime}(z)$. The behavior of $A i(z)$ at infinity follows from the asymptotic formulas,

$$
\begin{aligned}
& A i(z)=\frac{1}{2} \pi^{-\frac{1}{2}} z^{-\frac{1}{t}} e^{-\zeta}\left[1+O\left(\zeta^{-1}\right)\right] \quad z \rightarrow \infty, \\
& -\pi<\arg z<\pi \\
& A i(-z)=\pi^{-\frac{1}{2}} z^{-1}\left[\cos \left(\zeta-\frac{\pi}{4}\right)+O\left(\zeta^{-1}\right)\right] \\
& z>0, \quad z \rightarrow \infty,
\end{aligned}
$$

where

$$
\zeta=\frac{2}{3} z^{\frac{3}{2}}
$$

The behavior of $B i(z)$ can be deduced by means of the relation,

$$
B i(z)=i\left[\omega^{2} A i\left(\omega^{2} z\right)-\omega A i(\omega z)\right] .
$$

It follows that the functions,

$$
\begin{array}{lll}
\left(1+|z|^{\frac{1}{5}}\right) A i(z) e^{5}, & \left(1+|z|^{\frac{1}{4}}\right)^{-1} A i^{\prime}(z) e^{5} & |\arg z| \leq \pi \\
\left(1+|z|^{\frac{1}{2}}\right) B i(z) e^{-5}, & \left(1+|z|^{\frac{1}{4}}\right)^{-1} B i^{\prime}(z) e^{-5} & |\arg z| \leq \pi / 3 \\
\left(1+|z|^{\frac{1}{4}}\right) B i(z) e^{5}, & \left(1+|z|^{\frac{1}{4}}\right)^{-1} B i^{\prime}(z) e^{5} & \pi / 3 \leq|\arg z| \leq \pi,
\end{array}
$$

and the reciprocals of the functions,

$$
\begin{aligned}
& \left(1+|z|^{\frac{1}{2}}\right)\left[|A i(z)|^{2}+|B i(z)|^{2}\right]^{\frac{1}{2}} e^{5} \\
& \left(1+|z|^{\frac{1}{2}}\right)^{-1}\left[\left|A i^{\prime}(z)\right|^{2}+\left|B i^{\prime}(z)\right|^{2}\right]^{\frac{1}{2}} e^{5} \quad \arg z= \pm \pi
\end{aligned}
$$

are bounded.

As a consequence we have the following:

\section{Lemma 1}

The following functions are bounded:

$$
\begin{gathered}
\Phi(x) Y_{m}(x) \exp \left[\sigma_{m}(x)\right], \quad\left\{\left[\Phi(x) \tilde{Y}_{m}(x) \exp \left[\sigma_{m}(x)\right]\right\}^{ \pm 1}\right. \\
\text { for } m=0, \pm 1 \text { and }\left|\arg \theta_{m}\right| \leq \pi \text { or } m=2 \text { and } \theta<0 \\
{\left[\Phi(x) Y_{2}(x) e^{-\sigma(x)}\right]^{ \pm 1} \text { for }|\arg \theta| \leq \pi / 3}
\end{gathered}
$$

There are also corresponding results involving the $\widetilde{Z}_{m}$ and the derivatives of the $Y_{m}$, but these will not be noted in detail (see [4], lemma 2).

Since $Y_{m}$ and $Y_{m^{\prime}}$ are linearly independent solutions of (2.1) when $m, m^{\prime}=0, \pm 1,2$ and $m \neq m^{\prime}$, the Green's function can be expressed as a linear combination of any pair of these, indeed,

$$
K(x, t)=\frac{1}{\Delta}\left[Y_{m}(x) Y_{m^{\prime}}(t)-Y_{m}(t) Y_{m^{\prime}}(x)\right],
$$

where

$$
\Delta=Y_{m}^{\prime}(t) Y_{m^{\prime}}(t)-Y_{m}(t) Y_{m^{\prime}}(t)
$$

is the Wronskian of $Y_{m}$ and $Y_{m^{\prime}}$, which is independent of $t$ and can be computed from the known values of $A i(0), A i^{\prime}(0), B i(0)$, and $B i^{\prime}(0)$.

For the Green's function, we need the following result: 


\section{Lemma 2}

If $m=0$ and $a<t \leq x<b$, or $m= \pm 1$ and $a<x \leq t<b$, or else $m=2$ and either $a<x \leq t \leq c$ or $c \leq t \leq x<b$, then

$$
\left|K(x, t) \widetilde{Y}_{m}(t)\right| \leq C \lambda^{-3}|\Phi(t)|^{-2}\left|\widetilde{Y}_{m}(x)\right|
$$

and

$$
\begin{aligned}
&\left|\frac{\partial}{\partial x}\left[\left|\phi^{\prime}(x)\right|^{\frac{1}{2}} K(x, t)\right] \tilde{Y}_{m}(t)\right| \\
& \leq C \lambda^{-3}|\Phi(t)|^{-2}\left|\tilde{Z}_{m}(x)\right| .
\end{aligned}
$$

We shall prove (3.6) for $m=0$. The proof of (3.7) and the discussion of the cases $m= \pm 1,2$ are similar.

We use (3.5) with $m=0, m^{\prime}=1$, say, obtaining

$$
K(x, t)=2 \pi \omega^{4} \lambda^{-\frac{2}{3}}\left[Y_{0}(x) Y_{1}(t)-Y_{0}(t) Y_{1}(x)\right]
$$

and consequently,

$$
\begin{aligned}
& \frac{1}{2 \pi} \lambda^{\jmath} \Phi^{2}(t)\left|K(x, t) \frac{\widetilde{Y}_{0}(t)}{\tilde{Y}_{0}(x)}\right| \leq\left|\frac{Y_{0}(x)}{\widetilde{Y}_{0}(x)}\right|\left|\Phi^{2}(t) \widetilde{Y}_{0}(t) Y_{1}(t)\right| \\
&+\left|\frac{Y_{1}(x)}{\tilde{Y}_{0}(x)}\right|\left|\Phi^{2}(t) Y_{0}(t) \widetilde{Y}_{0}(t)\right| .
\end{aligned}
$$

Here, we take $\arg \theta=0$ and $\arg \theta_{1}=2 \pi / 3$ if $x<c$; and $\arg \theta=-\pi$ and $\arg \theta_{1}=-\pi / 3$ if $x>c$; noting that in either case $\sigma_{1}=-\sigma$, and $\operatorname{Re} \sigma(x)$ is a decreasing function of $x$. Clearly, $\left|Y_{0}(x) / \widetilde{Y}_{0}(x)\right| \leq 1$, and $\Phi^{2}(t) \widetilde{Y}_{0}(t) Y_{1}(t)$ is bounded by lemma 1 . Thus, the first term on the right hand side of (3.8) is bounded. Furthermore,

$$
\frac{Y_{1}(x)}{\widetilde{Y}_{0}(x)} e^{-2 \sigma(x)} \text { and } \Phi^{2}(t) Y_{0}(t) \widetilde{Y}_{0}(t) e^{2 \sigma(t)}
$$

are bounded (lemma 1), and $\left|e^{2 \sigma(x)-2 \sigma(t)}\right| \leq 1$ since $t \leq x$ and $\operatorname{Re} \sigma(x)$ is a decreasing function of $x$. Thus, the second term on the right hand side of (3.8) is also bounded, and (3.6) is proved in this case.

\section{The Integral Equation and Its Solution}

We shall now solve the integral equation (2.14) by the method of successive approximations. Under assumptions (i) to (iii) of Sec. 2, we fix $m$ and $\lambda$, and set

$$
\begin{gathered}
u_{0}(x)=Y_{m}(x) \\
u_{n+1}(x)=\int_{x_{m}}^{x} K(x, t) F(t, \lambda) u_{n}(t) d t \quad n=0,1,2, \cdots
\end{gathered}
$$

In order to prove the convergence of $\sum u_{n}(x)$, we first remark that according to assumption (iii) and lemma 2,

$$
\begin{array}{r}
\left|K(x, t) F(t, \lambda) \tilde{Y}_{m}(t)\right| \leq(C / \lambda)\left|\widetilde{Y}_{m}(x)\right||G(t, \lambda)| \\
\leq(C \Lambda / \lambda)\left|\widetilde{Y}_{m}(x)\right| g(t)
\end{array}
$$

Next, we prove by induction that

$$
\begin{array}{r}
\left|u_{n}(x)\right| \leq 1 /(n !)\left((C \Lambda / \lambda)\left|\int_{x_{m}}^{x} g(t) d t\right|\right)^{n}\left|\widetilde{Y}_{m}(x)\right| \\
\quad n=0,1,2, \cdots .
\end{array}
$$

Indeed, (4.4) is true for $n=0$. If it is true for any $n$, then it follows from $(4.2),(4.3),(4.4)$

$$
\begin{aligned}
\left|u_{n+1}(x)\right| \leq \frac{1}{n !}\left(\frac{C \Lambda}{\lambda}\right)^{n+1} & \\
& \times\left.\left|\tilde{Y}_{m}(x)\right|\left|\int_{x_{m}}^{x} g(t)\right| \int_{x_{m}}^{t} g(s) d s\right|^{n} d t \mid .
\end{aligned}
$$

Since

$$
\int_{x_{m}}^{x} g(t)\left[\int_{x_{m}}^{t} g(s) d s\right]^{n} d t=(n+1)^{-1}\left[\int_{x_{m}}^{x} g(s) d s\right]^{n+1},
$$

this proves (4.4) for $n+1$, and hence for all $n$.

After proving (4.4), we note that, by assumption (iii), $\Lambda(\lambda) / \lambda$ is a bounded function of $\lambda$ for $\lambda \geq \lambda_{0}>0$, and

$$
\int_{x_{m}}^{x} g(t) d t
$$

is a bounded function of $x$, so that

$$
\left|u_{n}(x)\right| \leq \frac{1}{n !} A^{n}\left|\widetilde{Y}_{m}(x)\right| \quad n=0,1,2, \cdots,
$$

where $A$ is independent of $x$ and $\lambda$. It follows that

$$
\sum u_{n}(x) / \widetilde{Y}_{m}(x)
$$

converges uniformly and absolutely for $a<x<b$, and

$$
y_{m}(x)=\sum_{n=0}^{\infty} u_{n}(x)
$$

defines a function which is clearly continuous for $a<x<b$, and satisfies (2.14). Also,

$$
\begin{aligned}
\left|y_{m}(x)\right| \leq \tilde{Y}_{m}(x) \mid \exp \left(\frac{C \Lambda}{\lambda}\left|\int_{x_{m}}^{x} g(t) d t\right|\right) & \\
& \leq\left|\widetilde{Y}_{m}(x)\right| e^{A} .
\end{aligned}
$$

The uniqueness of the solution can be proved in the usual manner.

To prove the differentiability of $y_{m}(x)$, we first form $d /(d x)\left[u_{n}(x) \phi^{\prime \frac{1}{2}}(x)\right]$

$$
=\int_{x_{m}}^{x} \partial /(\partial x)\left[K(x, l) \phi^{\prime \prime}(x)\right] F(t, \lambda) u_{n-1}(t) d t .
$$

Here, we use assumption (iii), lemma 2, and (4.4) to 
show that

$$
\left|\frac{d}{d x}\left[u_{n}(x) \phi^{\prime t}(x)\right]\right| \leq \frac{1}{n !}\left(\frac{C \Lambda}{\lambda}\left|\int_{x_{m}}^{x} g(t) d t\right|\right)^{n}\left|Z_{m}(x)\right|,
$$

and conclude that the series

$$
\sum\left(u_{n} \phi^{\prime \prime}\right)^{\prime} / \widetilde{Z}_{m}(x)
$$

converges uniformly, and hence that $\sum u_{n} \phi^{\prime \frac{1}{3}}$ may be differentiated term by term to yield $\left(y_{m} \phi^{\prime}\right)$. Finally,

$$
y_{m}^{\prime}(x)=\phi^{\prime-\mathrm{t}}\left[y_{m}(x) \phi^{\prime \prime}(x)\right]^{\prime}-\frac{\phi^{\prime \prime}}{\phi^{\prime}} y_{m}(x)
$$

is continuous for $a<x<b$.

Next, we remark that it is seen from (4.2) that

$$
\begin{aligned}
u_{n}^{\prime \prime}(x) & =F(x, \lambda) u_{n-1}(x)+\int_{x_{m}}^{x} \frac{\partial^{2}}{\partial x^{2}} K(x, t) F(t, \lambda) u_{n-1}(t) d t \\
& =F(x, \lambda) u_{n-1}(x)-\left[\lambda^{2} \phi \phi^{\prime 2}+\frac{1}{2}\{\phi, x\}\right] u_{n}(x)
\end{aligned}
$$

since $K(x, t)$ satisfies (2.4). As before, it follows that (4.6) may be differentiated a second time, $y_{m}{ }^{\prime \prime}(x)$ is continuous, and $y_{m}(x)$ satisfies (2.4) for $a<x<b$.

To estimate $y_{m}-Y_{m}$, we use (2.14), (4.7), and (4.3).

$$
\begin{aligned}
\left|y_{m}(x)-Y_{m}(x)\right| \leq e^{A}\left|\int_{x_{m}}^{x}\right| K(x, t) F(t, \lambda) \widetilde{Y}_{m}(t)|d t| \\
\quad \leq \frac{C \Lambda}{\lambda} e^{A}\left|\widetilde{Y}_{m}(x)\right|\left|\int_{x_{m}}^{x} g(t) d t\right| .
\end{aligned}
$$

This completes the proof.

\section{Application to Bessel Functions}

We shall now apply our results to Bessel functions of large order. The asymptotic forms of these functions were determined from the differential equation they satisfy by Langer, Cherry, Olver, and also in [3], Sec. 4.8. Here, we shall show that the leading term of the asymptotic expansion follows from our result without much effort.

The functions,

$$
x^{ \pm} J_{\lambda}(\lambda x), \quad x^{\frac{1}{3}} H_{\lambda}^{(1)}(\lambda x), \quad x^{\frac{1}{3} H_{\lambda}^{(2)}}(\lambda x),
$$

satisfy the differential equation,

$$
\frac{d^{2} y}{d x^{2}}+\left[\lambda^{2}\left(1-\frac{1}{x^{2}}\right)+\frac{1}{4 x^{2}}\right] y=0 .
$$

This equation is of the form (1.1) and it will turn out that all our conditions are satisfied on the interval $0<x<\infty$. According to (2.3) we set

$$
\phi \phi^{\prime 2}=1-\left(1 / x^{2}\right) \text {. }
$$

and obtain by integration

$$
\begin{aligned}
& \frac{2}{3}[-\phi(x)]^{\frac{k}{2}}=\alpha(x)=\int_{x}^{1}\left(\frac{1}{t^{2}}-1\right)^{\frac{1}{2}} d t \\
& =-\left(1-x^{2}\right)^{\frac{1}{2}}+\log \left[1+\left(1-x^{2}\right)^{1}\right]-\log x \\
& \begin{array}{rlr}
\frac{2}{3}[\phi(x)]^{\frac{1}{2}}=\beta(x) & =\int_{1}^{x}\left(1-\frac{1}{t^{2}}\right)^{\frac{1}{2} d t} & 0<x \leq 1 \\
& =\left(x^{2}-1\right)^{\frac{1}{2}-\cos ^{-1} \frac{1}{x}} 1 \leq x<\infty .
\end{array}
\end{aligned}
$$

We note that

$$
\begin{array}{ll}
\alpha(x)=-\log x+\log 2-1+O\left(x^{2}\right) & \text { as } x \rightarrow 0 \\
\alpha^{\prime}(x)=-\frac{1}{x}+O(x) & \text { as } x \rightarrow 0 \\
\beta(x)=x-\frac{\pi}{2}+O(1 / x) & \text { as } x \rightarrow \infty, \\
\beta^{\prime}(x)=1+O\left(1 / x^{2}\right) & \text { as } x \rightarrow \infty .
\end{array}
$$

The differential equation (5.2) may now be written in the form (2.4) with

$$
F(x, \lambda)=F(x)=\frac{1}{2}\{\phi, x\}-1 /\left(4 x^{2}\right) .
$$

We proceed to verify that the conditions (i) to (iii) of Sec. 2 are satisfied. Clearly, $\phi(x)$ is three times continuously differentiable (indeed it is analytic). Also $\phi^{\prime}(x)>0$, and it follows that $F(x)$ is continuous (in fact, analytic). We also have

$$
|G(t, \lambda)|=\left|\frac{\lambda^{\ddagger} F(t)}{\Phi^{2}(t)}\right| \leq \frac{|F(t)|}{|\phi(t)|^{\mid} \phi^{\prime}(t)}=\frac{t|F(t)|}{\left|1-t^{2}\right|^{\prime}}
$$

and in order to verify assumption (iii), we shall investigate the behavior of $F(t)$ as $t \rightarrow 0$ and as $t \rightarrow \infty$. To do this, we use the chain rule

$$
\{\phi, x\}=\{\phi, \chi\} \chi^{\prime 2}+\{\chi, x\}
$$

for the Schwarzian derivative, setting $\chi=\alpha$ when $0<x<1$, and $\chi=\beta$ when $1<x<\infty$. Thus,

$F(x)=\frac{5 \alpha^{\prime 2}}{18 \alpha^{2}}+\frac{1}{2}\{\alpha, x\}-\frac{1}{4 x^{2}}=\frac{5 \alpha^{\prime 2}}{18 \alpha^{2}}-\frac{4+x^{2}}{4\left(1-x^{2}\right)^{2}}$

$F(x)=\frac{5 \beta^{\prime 2}}{18 \beta^{2}}-\frac{4+x^{2}}{4\left(1-x^{2}\right)^{2}} \quad 1<x<\infty$.

$0<x<1$

From these formulas, in conjunction with (5.6) and (5.7), it is seen that

$$
\begin{array}{lll}
F(x)=O\left[(x \log x)^{-2}\right] & \text { as } & x \rightarrow 0 \\
F(x)=O\left(x^{-2}\right) & \text { as } & x \rightarrow \infty
\end{array}
$$


It follows that

$$
\left[x \log \left(2+\frac{1}{x}\right)\right]^{2} F(x)
$$

is bounded when $0<x<\infty$, and we may set

$$
g(t)=\left[t\left|1-t^{2}\right|^{\frac{1}{3}} \log ^{2}\left(2+\frac{1}{t}\right)\right]^{-1}
$$

and $\Lambda$ some fixed number (independent of $t$ and $\lambda$ ) in assumption (iii). Clearly

$$
\int_{0}^{\infty} g(t) d t<\infty
$$

and we note in passing that

$$
\begin{aligned}
& \int_{0}^{x} g(t) d t=O(1 / \log x) \text { as } x \rightarrow 0 \\
& \int^{\infty} g(t) d t=O(1 / x) \quad \text { as } \quad x \rightarrow \infty .
\end{aligned}
$$

On verifying all assumptions, we know that there exist solutions $y_{m}(x)$ of (5.2) for which (2.15) holds. It remains to identify these solutions in terms of the functions (5.1).

Let us start with $y_{0}(x)$. By fixing $\lambda>0$, and making $x \rightarrow 0$, we have from (2.15) and (5.14),

$$
y_{0}(x)=\phi^{\prime-1} A i\left(-\lambda^{3} \phi\right)\left[1+O\left(\frac{1}{\log x}\right)\right] \text { as } x \rightarrow 0 .
$$

Since $-\phi \rightarrow \infty$ as $x \rightarrow 0$, we may use (3.1) and (5.6) to write

$$
y_{0}(x)=\frac{\exp [-\lambda \alpha(x)]}{2\left(\pi \phi^{\prime}\right)^{1}(-\phi)^{1}}\left[1+O\left(\frac{1}{\log x}\right)\right] \text { as } x \rightarrow 0 \text {. }
$$

Here, we use (5.6) and (5.3) to obtain the asymptotic behavior of $y_{0}(x)$ as $x \rightarrow 0, \lambda$ being fixed.

$$
\begin{aligned}
y_{0}(x)=\frac{1}{2} \pi^{-\frac{1}{2}} 2^{-\lambda} \lambda^{-1 / 6} e^{\lambda} x^{\lambda+1} & \\
& \times\left[1+o\left(\frac{1}{\log x}\right)\right] \text { as } x \rightarrow 0
\end{aligned}
$$

In order to avoid inessential complications, let us assume that $\lambda$ is not an integer, so that $J_{\lambda}$ and $J_{-\lambda}$ are linearly independent. Then, $y_{0}(x)$, being a solution of (5.2), must be of the form,

Now,

$$
y_{0}(x)=x:\left[c_{1}(\lambda) J_{\lambda}(\lambda x)+c_{2}(\lambda) J_{-\lambda}(\lambda x)\right] .
$$

$$
J_{ \pm \lambda}(\lambda x)=\frac{(\lambda x / 2)^{ \pm \lambda}}{\Gamma(1 \pm \lambda)}[1+O(x)] \text { as } x \rightarrow 0
$$

and substituting in (5.16) the asymptotic form of each term as $x \rightarrow 0$, we see that

$$
c_{1}(\lambda)=\frac{1}{2} \pi^{-1} \lambda^{-\lambda-1 / 6} e^{\lambda} \Gamma(\lambda+1), \quad c_{2}(\lambda)=0,
$$

so that

We then have

$$
x^{\mathrm{j}} J_{\lambda}(\lambda x)=\frac{2 \pi^{\ddagger}}{\Gamma(\lambda+1)} e^{-\lambda} \lambda^{\lambda+1 / 6} y_{0}(x) .
$$

$$
\begin{aligned}
& J_{\lambda}(\lambda x)=\frac{2 \pi^{\frac{1}{2}}}{\Gamma(\lambda+1)} e^{-\lambda} \lambda^{\lambda+1 / 6}\left(x \phi^{\prime}\right)^{-1} A i\left(-\lambda^{\prime} \phi\right) \\
& \times\left[1+O\left(\frac{1}{\lambda}\right)\right]
\end{aligned}
$$

for $\lambda>0$ and $0<x<\infty$, except that in case $x>1$, the error term must be modified in order to take account of the zeros of $A i$. When $x<1, O\left(\lambda^{-1}\right)$ in (5.18) may be replaced by $O\left[(\lambda \log x)^{-1}\right]$. The asymptotic form of the derivative may be obtained similarly.

A somewhat simpler asymptotic form results if Stirling's formula is used for $\Gamma(\lambda+1)$.

$$
J_{\lambda}(\lambda x)=\left(\frac{1}{2} \lambda^{i} x \phi^{\prime}\right)^{-\frac{1}{1}} A i\left(-\lambda^{\frac{1}{3}} \phi\right)\left[1+O\left(\lambda^{-1}\right)\right]
$$

for $\lambda>0$ and $0<x<\infty$, with the same modification of the error term near zeros of $A i\left(-\lambda^{2} \phi\right)$ as before. In (5.19) the $O$ symbol cannot be strengthened for $0<x<1$.

We now proceed to $y_{1}(x)$, fix $\lambda>0$, and make $x \rightarrow \infty$. From (2.15) and (5.14),

$$
y_{1}(x)=\frac{1}{\phi^{\prime-\frac{1}{2}}} A i\left(-\lambda^{\frac{2}{\omega} \omega \phi}\right)\left[1+O\left(\frac{1}{x}\right)\right] \text { as } x \rightarrow \infty .
$$

Since $\phi \rightarrow \infty$ as $x \rightarrow \infty$, we may use (3.1) and (5.7)

$$
\begin{aligned}
& y_{1}(x)\left.\left.=\frac{\exp [i \lambda \beta(x)+i \pi / 12]}{2\left(\pi \phi^{\prime}\right)^{\frac{1}{4}}}\right] 1+o\left(\frac{1}{x}\right)\right] \text { as } x \rightarrow \infty \\
&=\frac{1}{2} \pi^{-i} \lambda^{-1 / 6} \exp \left[i\left(\lambda x-\frac{\lambda \pi}{2}+\frac{\pi}{12}\right)\right]\left[1+o\left(\frac{1}{x}\right)\right] \\
& \text { as } x \rightarrow \infty .
\end{aligned}
$$

$y_{1}(x)$, being a solution of (5.2), must be of the form,

$$
y_{1}(x)=x^{3}\left[c_{3}(\lambda) H_{\lambda}^{(1)}(\lambda x)+c_{4}(\dot{\lambda}) H_{\lambda}^{(2)}(\lambda x)\right] \text {. }
$$

We know that

$$
\begin{aligned}
x^{1} H_{\lambda}^{(1,2)}(\lambda x)=\left(\frac{2}{\pi \lambda}\right)^{i} \exp [ & \left. \pm i\left(\lambda x-\frac{\lambda \pi}{2}-\frac{\pi}{4}\right)\right] \\
& \times\left[1+o\left(\frac{1}{x}\right)\right] \text { as } x \rightarrow \infty,
\end{aligned}
$$

where + is for $H_{\lambda}^{(1)}$ and - for $B_{\lambda}^{(2)}$, and conclude, similarly to the previous case, that

$$
x^{\mathfrak{t}} H_{\lambda}^{(1)}(\lambda x)=2^{\mathfrak{l} \lambda} \lambda^{-1} e^{-\pi i / 3} y_{1}(x),
$$


and hence

$$
\begin{aligned}
H_{\lambda}^{(1)}(\lambda x)=2^{\frac{b}{2}} \lambda^{-\frac{1}{2}} e^{-\pi i / 3}\left(x \phi^{\prime}\right)^{-\frac{1}{2}} A i( & \left.-\lambda^{i} \omega \phi\right) \\
& \times\left[1+O\left(\frac{1}{\lambda}\right)\right]
\end{aligned}
$$

for $\lambda>0$ and $0<x<\infty$. If $x>1, O\left(\lambda^{-1}\right)$ may be replaced by $O\left[(\lambda x)^{-1}\right]$. The corresponding asymptotic form for the second Hankel function for $\lambda>0$ and $0<x<\infty$ is

$$
\begin{aligned}
H_{\lambda}{ }^{(2)}(\lambda x)=2^{3} \lambda^{-1} e^{i \pi / 3}\left(x \phi^{\prime}\right)^{-1} A i\left(-\lambda^{3} \omega^{-1} \phi\right) & \\
\times & {\left[1+O\left(\frac{1}{\lambda}\right)\right] }
\end{aligned}
$$

with the same remarks about the error term as before.

\section{Application to Hermite Polynomials}

Next we shall apply our results to Hermite polynomials of large order. The asymptotic forms of these have been the subject of several investigations. For references and a summary of results see [21]. Since Hermite polynomials are connected with parabolic cylinder functions, results regarding the latter functions are also relevant [8], [11]. Here, we shall follow the discussion given by $\mathbf{H}$. Skovgaard [19].

$n$ is a nonnegative integer, and $N=2 n+1$ throughout this section. It is known [7, Vol. II, p. $193 \mathrm{Eq}$. (13)] that

$$
\exp \left(-\frac{1}{2} N x^{2}\right) H_{n}\left(N^{1} x\right)
$$

satisfies the differential equation,

$$
\frac{d^{2} y}{d x^{2}}+N^{2}\left(1-x^{2}\right) y=0,
$$

whose general solution may also be written in the form,

$$
c_{1} D_{n}\left[(2 N)^{\frac{1}{x}} x\right]+c_{2} D_{-n-1}\left[(2 N)^{\frac{1}{3}} x\right] .
$$

(6.2) is of the form (1.1) on $(a, \infty)$, where $-1<a<0$, and $N$ takes the place of $\lambda$. Since $p(x)$ changes here from positive to negative values as $x$ increases, we shall have $\phi^{\prime}(x)<0$ and will use the second form of the results summarized in Sec. 2. Accordingly, we set

and obtain

$$
\phi \phi^{\prime 2}=1-x^{2}
$$

$$
\begin{aligned}
\frac{2}{3} \phi^{3}=\alpha(x) & =\int_{x}^{1}\left(1-t^{2}\right)^{\frac{1}{3}} d t \\
= & -\frac{1}{2} x\left(1-x^{2}\right)^{\frac{1}{3}}+\frac{1}{2} \cos ^{-1} x \quad a<x \leq 1, \\
\frac{2}{3}(-\phi)^{3}=\beta(x) & =\int_{1}^{x}\left(t^{2}-1\right)^{\frac{1}{d}} d t \\
= & \frac{1}{2} x\left(x^{2}-1\right)^{\frac{1}{2}-\frac{1}{2}} \cosh ^{-1} x \quad 1 \leq x<\infty,
\end{aligned}
$$

where $\cos ^{-1}$ and $\cosh ^{-1}$ denote the principal branches of the inverse functions. We then have (2.4) with

$$
\begin{aligned}
& F(x, \lambda)=F(x)=\frac{1}{2}\{\phi, x\} \\
&=\frac{5}{16} \frac{1-x^{2}}{\phi^{3}}-\frac{3 x^{2}+2}{4\left(1-x^{2}\right)^{2}} \quad x \neq 1,
\end{aligned}
$$

and, if we define $F(1)=\lim F(x)$ as $x \rightarrow 1, F(x)$ is continuous for $a \leq x<\infty$. Moreover, it can be shown that $(1+x)^{2} F(x)$ is bounded on $(a, \infty)$. Thus all the conditions of Sec. 2 will be satisfied if we take $\Lambda(\lambda)$ to be some suitable number independent of $x$ and $\lambda$, and set

$$
g(x)=(1+x)^{-2}\left|1-x^{2}\right|^{-1}
$$

Since $g(x)=O\left(x^{-3}\right)$ as $x \rightarrow \infty$, we also have

$$
\int_{x}^{\infty} g(t) d t=O\left(\frac{1}{1+x^{2}}\right) \quad a<x<\infty .
$$

We now apply the results of Sec. 2 with $m=0, N$ fixed, and $x \rightarrow \infty$. Since $-\phi(x)>0$ in this case, $\widetilde{Y}_{0}=Y_{0}$ and we have from (6.8)

$$
y_{0}(x)=\left|\phi^{\prime}\right|-\frac{1}{A} A i\left(-N^{z} \phi\right)\left[1+O\left(\frac{1}{1+x^{2}}\right)\right] \text {. }
$$

Since $-\phi \rightarrow \infty$, we use (3.1) to obtain

$$
y_{0}(x)=\frac{\exp [-N \beta(x)]}{2 \pi^{\frac{1}{2}} N^{1 / 6}\left(-\phi \phi^{\prime 2}\right)^{\frac{1}{2}}}\left[1+O\left(x^{-2}\right)\right] \text { as } x \rightarrow \infty \text {. }
$$

From (6.5)

$$
\beta(x)=\frac{1}{2} x^{2}-\frac{1}{2} \log (2 x)-\frac{1}{4}+O\left(x^{-2}\right) \quad x \rightarrow \infty
$$

and hence,

$$
\begin{aligned}
y_{0}(x)=\frac{(2 x)^{n}}{(2 \pi)^{1} N^{1 / 6}} \exp \left[-\frac{1}{2} N\left(x^{2}-\frac{1}{2}\right)\right] \\
\times\left[1+O\left(x^{-2}\right)\right] \quad x \rightarrow \infty
\end{aligned}
$$

In particular, it is seen that $y_{0}(x) \rightarrow 0$ as $x \rightarrow \infty$. $y_{0}(x)$, being a solution of (6.2) is of the form (6.3). Now, in (6.3), the first term vanishes, and the second term is unbounded, as $x \rightarrow \infty[7$, vol. II p. 122, Eq. (1)]. It follows that $c_{2}=0$ and $y_{0}(x)$ is a constant multiple of (6.1). From the explicit formula for Hermite polynomials [7, vol II, p. 193, Eq. (9)],

$$
\begin{aligned}
& \exp \left(-\frac{1}{2} N x^{2}\right) H_{n}\left(N^{1} x\right) \\
&=\left(2 N^{1} x\right)^{n} \exp \left(-\frac{1}{2} N x^{2}\right)\left[1+O\left(x^{-2}\right)\right]
\end{aligned}
$$

as $x \rightarrow \infty$ and a comparison of (6.9), and (6.10) shows that

$$
H_{n}\left(N^{1} x\right)=(2 \pi)^{\frac{1}{3}} N^{n / 2+1 / 6} \exp \left[\frac{1}{2} N\left(x^{2}-\frac{1}{2}\right)\right] y_{0}(x) .
$$

If we combine this result with (2.15), we obtain the 
asymptotic formula,

$$
\begin{aligned}
H_{n}\left(N^{1} x\right) & =(2 \pi)^{\frac{1}{2}} N^{n / 2+1 / 6} \exp \left[\frac{1}{2} N\left(x^{2}-\frac{1}{2}\right)\right] \\
\times\left|\phi^{\prime}\right|^{-\frac{3}{3}} A i\left(-N^{3} \phi\right) & {\left[1+O\left(\frac{1}{n\left(1+x^{2}\right)}\right)\right], }
\end{aligned}
$$

which is valid uniformly in $-1<a<x<\infty$ as $n \rightarrow \infty$, except that the error term must be modified to take care of the zeros of the Airy function when $a<x<1$. This result can be extended to $-\infty<x<0$ by using $H_{n}(-x)$ $=(-1)^{n} H_{n}(x)$.

Simpler asymptotic formulas holding for restricted ranges of $x$ may be deduced from (6.11). In [19] all the known formulas are so deduced, in part with improved conditions of validity. Here, we shall restrict ourselves to a brief indication of two of these results. have

If we take $0 \leq x<1$ and set $x=\cos \theta, 0<\theta \leq \pi / 2$, we

$$
\alpha=\frac{1}{4}(2 \theta-\sin 2 \theta)
$$

from (6.4). By using (3.2) in (6.11) we then obtain $H_{n}\left(N^{\frac{1}{3}} \cos \theta\right)$

$=2^{\frac{1}{2}} N^{n / 2} \sin ^{-\frac{1}{2}} \theta \exp \left(\frac{1}{4} N \cos 2 \theta\right)$

$$
\times\left\{\cos \left[\frac{1}{4} N(2 \theta-\sin 2 \theta)-\frac{1}{4} \pi\right]+O\left(n^{-1} \theta^{-3}\right)\right\} .
$$

For $x>1$, we set $x=\cosh \theta, \theta>0$, and obtain similarly

$$
\begin{array}{r}
H_{n}\left(N^{\frac{1}{3}} \cosh \theta\right)=(2 \sinh \theta)^{-\frac{1}{1}} N^{n / 2} \exp \left[\frac{1}{4} N\left(2 \theta+e^{-2 \theta}\right)\right] \\
\times\left[1+O\left(n^{-1} \sinh ^{-3} \frac{2 \theta}{3}\right)\right] .
\end{array}
$$

\section{SINGULARITIES}

\section{Outline of the Method and Results}

Here, we shall give only a brief outline of the method following Swanson and state the result. The proofs are similar to those given in Sec. 3 and 4 for the transition point case and can be found in [20]. By using (1.4) as our comparison equation, we introduce a new variable $x$ by the substitution,

$$
t=\psi(x), \quad w=\left|\psi^{\prime}(x)\right|^{1} U,
$$

thus changing (1.4) into

$$
\frac{d^{2} U}{d x^{2}}+\left[\left(\frac{\lambda^{2}}{\psi}+\frac{1-\nu^{2}}{4 \psi^{2}}\right) \psi^{\prime 2}+\frac{1}{2}\{\psi, x\}\right] U=0,
$$

an equation whose solutions are known from (1.5). $\{\psi, x\}$ is defined by (2.2). The differential equations (1.1) and (7.1) will be nearly equal for large $\lambda$ if we choose $\psi$ so that

$$
\psi^{-1} \psi^{\prime 2}=p(x)
$$

With this choice of $\psi,(1.1)$ may be written in the form,

$$
\frac{d^{2} y}{d x^{2}}+\left[\left(\frac{\lambda^{2}}{\psi}+\frac{1-\nu^{2}}{4 \psi^{2}}\right) \psi^{\prime 2}+\frac{1}{2}\{\psi, x\}\right] y=F(x, \lambda) y,
$$

where

$$
F(x, \lambda)=\frac{1-\nu^{2}}{4 \psi^{2}} \psi^{\prime 2}+\frac{1}{2}\{\psi, x\}-r(x, \lambda) .
$$

As in the case of a transition point, we shall assume that the differential equation has been transformed to this form and, moreover, that $\nu$ has been so chosen that $|p(x)|^{-\frac{1}{2}} F(x, \lambda)$ is an integrable function of $x$ for each $\lambda>0$.

As a typical case, let us assume that $(x-c) p(x)$ is a twice continuously differentiable function of $x$ and $(x-c)^{2} r(x, \lambda)$, for each $\lambda>0$, a continuously differentiable function of $x$, for $a<x<b$ so that

$$
\begin{aligned}
p(x) & =q_{0}(x-c)^{-1}[1+O(x-c)] \\
r(x, \lambda) & =r_{0}(x-c)^{-2}[1+O(x-c)] \text { as } x \rightarrow c
\end{aligned}
$$

and assume $q_{0}>0, r_{0} \leq \frac{1}{4}, r_{0}$ independent of $\lambda$. Then $\psi(x)=q_{0}(x-c)[1+O(x-c)]$ from $(7.2), \psi^{\prime}(x) / \psi(x)$ $=(x-c)^{-1}[1+O(x-c)],\{\psi, x\}=O\left[(x-c)^{-1}\right]$ and

$$
F(x, \lambda)=\frac{1-v^{2}-4 r_{0}}{4(x-c)^{2}}+O\left[(x-c)^{-1}\right] \quad x \rightarrow c,
$$

and the integrability condition is satisfied if and only if $\nu=\left(1-4 r_{0}\right)^{2}$.

If $F(x, \lambda)=0,(7.3)$ is identical with (7.1). In general, we obtain from (7.3) by the method of variation of parameters, the integral equation of Volterra type,

$$
y(x)=U(x)+\int_{x^{\prime}}^{x} K(x, t) F(t, \lambda) y(t) d t
$$

satisfied by solutions of (7.3). Here, $U$ is a solution of (7.1), $x^{\prime}$ is a fixed point of the interval, and $K(x, t)$ is the Green's function of (7.1). For a fixed $t$ in $(a, b), K(x, t)$, considered as a function of $x$, satisfies (7.1) for $x \neq t$, and also satisfies the initial conditions $K(t, t)=0$, $(\partial K / \partial x)(t, t)=1$.

The significant solutions of (7.1) are obtained from (1.5) as

$$
\begin{aligned}
U_{0}(x) & =\left(\psi / \psi^{\prime}\right)^{\frac{1}{2}} J_{\nu}\left(2 \lambda \psi^{\frac{1}{1}}\right), \\
U_{1}(x) & =\left(\psi / \psi^{\prime}\right)^{\frac{1}{2}} H_{\nu}^{(1)}\left(2 \lambda \psi^{\frac{1}{2}}\right), \\
U_{-1}(x) & =\left(\psi / \psi^{\prime}\right)^{\frac{1}{2}} H_{\nu}^{(2)}\left(2 \lambda \psi^{\frac{1}{2}}\right) .
\end{aligned}
$$

For the error estimates, it is desirable to modify $U_{0}$ in the region where it has zeros. Let $\Delta>0$ be so chosen that $J_{\nu}(z)>0$ for $0<z \leq \Delta$, and set

$$
\begin{aligned}
\tilde{U}_{0}(x) & =U_{0}(x) \text { if } 4 \lambda^{2} \psi \leq \Delta^{2}, \\
& =\left(\psi / \psi^{\prime}\right)^{\frac{1}{2}}\left[\left|J_{\nu}\left(2 \lambda \psi^{3}\right)\right|^{2}+\left|Y_{\nu}\left(2 \lambda \psi^{3}\right)\right|^{2}\right]^{\frac{1}{2}} \\
\tilde{U}_{ \pm_{1}}(x) & =U_{ \pm_{1}}(x) .
\end{aligned}
$$

It is known from the theory of Bessel functions that the functions

$$
\begin{aligned}
& z^{y}\left(1+|z|^{1+\nu}\right) e^{-|\operatorname{Im} z|} J_{\nu}(z) \\
& z^{y}\left(1+|z|^{1-\nu}\right) e^{\mp i z} H_{\nu}^{(1,2)}(z)
\end{aligned}
$$


are bounded when $|\operatorname{argz}| \leq \pi$, except that a slight modification of the second function is necessary when $\nu=0$ and $H_{0}^{(1,2)}(z)$ has a logarithmic singularity. Also, in the second function the upper sign is taken for $H_{\nu}{ }^{(1)}$ and the lower sign for $B_{\nu}{ }^{(2)}$. Moreover, the second function has a bounded reciprocal.

This information can be used to develop results which correspond to lemma 1 and give bounds for the $U_{m}$, $U_{m}{ }^{\prime}, U_{m}, 1 / \tilde{U}_{m}$. These results are in turn used to prove under certain conditions [see (iv) below],

$$
\left|K(x, t) \tilde{U}_{m}(t)\right| \leq C \lambda^{-1}|p(x)|^{-t}\left|\widetilde{U}_{m}(x)\right|,
$$

together with a corresponding estimate for $\partial K(x, t) / \partial x$; in fact, to establish an analogue of lemma 2 in this case.

From here on, the investigation resembles that described in Sec. 4. The integral equation is solved by successive approximations, and the uniqueness and differentiability of the solution is proved. The estimates arising out of the successive approximations are used under the integral sign to provide a better estimate of $y(x)-U(x)$. There is one new feature, though. $x=c$ is a singular point of the integral equation and it is impossible to continue a general solution of (7.3) across this point. Accordingly, solutions corresponding to $x^{\prime}=a$ or $x^{\prime}=b$ exist only on the intervals $(a, c)$ or $(c, b)$, respectively. Again, for $x^{\prime}=c$, the integral equation has a solution only if $U(x)$ in (7.5) is a multiple of $U_{0}(x)$. This solution can be continued across $x=c$, but the continuation is not unique: indeed, in $U_{0}(x)$ itself we may take $\arg \psi t=\pi / 2$, or $\arg \psi t=-\pi / 2$ when $x<c$.

Apart from this, the discussion of the integral equation resembles the discussion given in Sec. 4 and leads to the following result:

If (i) $\psi(x)$ is three times continuously differentiable and $\psi^{\prime}(x)>0$ for $a<x<b$, and $\psi(c)=0$; (ii) for each fixed $\lambda>0, F(x, \lambda)$ is a continuous function of $x$ for $a<x<c$ and $c<x<b$; (iii) $|p(x)|^{-1}|F(x, \lambda)| \leq \Lambda(\lambda) g(x)$ for $x \neq c$ where $(1+\lambda)^{-1} \Lambda(\lambda)$ is a bounded function of $\lambda$ and $g(x)$ is integrable over $(a, b)$; and (iv) either $m=0, x_{0}=c$, and $a<x<b$ or $m= \pm 1, x_{m}=a, \arg \phi=m \pi$, and $a<x<c$ or $m= \pm 1, x_{m}=b, \arg \phi=0$, and $c<x<b ;$ then the integral equation,

$$
u_{m}(x)=U_{m}(x)+\int_{x_{m}}^{x} K(x, t) F(t, \lambda) u_{m}(t) d t
$$

has a unique solution for each $\lambda>0$; this solution is twice continuously differentiable and satisfies (7.3), except possibly for $x=c$ in case $m=0$, and on the intervals specified in (iv),

$$
u_{m}(x)=U_{m}(x)+O\left[\frac{\Lambda(\lambda)}{\lambda} \widetilde{U}_{m}(x) \int_{x_{m}}^{x} g(t) d t\right] .
$$

There is a corresponding formula for $y_{m}{ }^{\prime}(x)$.

\section{Application to Laguerre Polynomials}

Laguerre polynomials of large order have been investigated by several writers. References and a sum- mary of results will be found in [21]. Of more recent investigations especially those by Tricomi [22] are noteworthy. Since these polynomials are connected with confluent hypergeometric functions, results regarding the latter functions are also pertinent; see [9] and the references given there.

Laguerre polynomials present an especially interesting feature in that the differential equation which they satisfy has both a singularity and a transition point. Accordingly, we shall obtain two asymptotic representations, one in terms of Bessel functions and the other in terms of Airy functions. The regions of validity of the two representations overlap, and in the overlapping part the two representations are asymptotically equivalent.

We shall consider Laguerre polynomials $L_{n}{ }^{\alpha}(z)$ with fixed $\alpha \geq 0$, large $n$, and unrestricted real $z$. Throughout this section, $N=n+(\alpha+1) / 2$. It is known $[7, \mathrm{Sec}$. 10.12] that

$$
x^{(\alpha+1) / 2} e^{-2 N x} L_{n}^{\alpha}(4 N x)
$$

satisfies the differential equation,

$$
\frac{d^{2} y}{d x^{2}}+\left[4 N^{2}\left(\frac{1}{x}-1\right)+\frac{1-\alpha^{2}}{4 x^{2}}\right] y=0
$$

Here, $N$ is the large parameter, and the differential equation has a singularity at $x=0$ and a transition point at $x=1$. We shall first study the asymptotic representation on an interval including the singularity.

From (7.2),

and we obtain

$$
\psi^{-1} \psi^{\prime 2}=4[(1 / x)-1]
$$

$$
\begin{aligned}
\psi(x) & =-\left[\left(x^{2}-x\right)^{\frac{1}{2}}+\sinh ^{-1}(-x)^{\frac{1}{2}}\right]^{2} & & x \leq 0, \\
& =\left[\left(x-x^{2}\right)^{\frac{1}{3}}+\sin ^{-1} x^{\frac{1}{1}}\right]^{2} & & 0 \leq x<1 .
\end{aligned}
$$

Here, all square roots are nonnegative, and $\sin ^{-1}, \sinh ^{-1}$ denote the principal branches of the respective functions. Also, $r(x, \lambda)=\left(1-\alpha^{2}\right) /\left(4 x^{2}\right), \quad r_{0}=\left(1-\alpha^{2}\right) / 4 \leq \frac{1}{4}$ here, and we obtain $\nu=\left(1-4 r_{0}\right)=\alpha$. (8.2) can then be written in the form (7.3) with

$$
F(x, \lambda)=F(x)=\frac{1-\alpha^{2}}{4 \psi^{2}} \psi^{\prime 2}+\frac{1}{2}\{\psi, x\}-\frac{1-\alpha^{2}}{4 x^{2}} \quad x \neq 0 .
$$

From the behavior of $\psi(x)$ near $x=0$ and $x=-\infty$, it is easy to show that $|x| F(x)$ is bounded in some neighborhood of $x=0$ and $x^{2} F(x)$, for say, $x \leq-1$. We can now verify conditions (i), (ii), (iii) of the preceding section on the interval $-\infty<x<b<1$, the last condition with $\Lambda(\lambda)$ a constant and $g(x)=[|x|(1-x)]^{-1}$ so that

$$
\int_{0}^{x} g(t) d t= \pm\left(\frac{|x|}{1-x}\right)^{1}
$$

We now apply the results of the preceding section with $m=0$. By fixing $N$ and making $x \rightarrow 0$, we have 
$\psi(x)=4 x[1+O(x)], \psi^{\prime}(x)=4+O(x)$ and from (7.9) in combination with the known behavior of $J_{\alpha}(z)$ near the origin,

$$
\begin{aligned}
u_{0}(x) & =\left(\frac{\psi}{\psi^{\prime}}\right)^{\frac{1}{2}} J_{\alpha}\left(2 N \psi^{\frac{1}{2}}\right)\left[1+O\left(|x|^{\left.\frac{1}{1}\right)}\right]\right. \\
& =\frac{(2 N)^{\alpha} x^{(\alpha+1) / 2}}{\Gamma(\alpha+1)}\left[1+O\left(|x|^{\left.\frac{1}{2}\right)}\right] \quad x \rightarrow 0 .\right.
\end{aligned}
$$

From the behavior of the general solution of (8.2) at $x=0$, it follows that (8.1) and $u_{0}(x)$ are numerical multiples of each other. Moreover, from the explicit representation of $L_{n}{ }^{\alpha}(z)$ [7, Vol. II, p. 188 Eq. (7)],

$$
\begin{aligned}
x^{(\alpha+1) / 2} e^{-2 N x} L_{n}{ }^{\alpha}(4 N x) & \\
= & \frac{\Gamma(n+\alpha+1)}{n ! \Gamma(\alpha+1)} x^{(\alpha+1) / 2}[1+O(x)] \quad x \rightarrow 0
\end{aligned}
$$

and from a comparison of (8.6) and (8.7),

$$
L_{n}{ }^{\alpha}(4 N x)=\frac{\Gamma(n+\alpha+1)}{n !(2 N)^{\alpha}} x^{-(\alpha+1) / 2} e^{2 N x} u_{0}(x) .
$$

From this formula in combination with (7.9), we obtain the asymptotic representation,

$$
\begin{aligned}
L_{n}{ }^{\alpha}(4 N x)= & \frac{\Gamma(n+\alpha+1)}{n !(2 N)^{\alpha}} x^{-(\alpha+1) / 2} e^{2 N x}\left(\frac{\psi}{\psi^{\prime}}\right)^{\prime} \\
& \quad \times J_{\alpha}\left(2 N \psi^{3}\right)\left[1+O\left(\frac{1}{N}\left|\frac{x}{1-x}\right|^{\frac{1}{3}}\right)\right]
\end{aligned}
$$

valid, with $N=n+(\alpha+1) / 2$ and $\psi$ given by (8.3), for $n=0,1,2, \cdots$ and $-\infty<x<b<1$, except that the error term must be modified in the vicinity of zeros of $J_{\alpha}$, which occur when $x>0$. For $x<0, \arg x=\arg \psi$ must be taken in (8.9) : with this convention, the right hand side of (8.9) is defined unambiguously.

We now turn to the transition point. According to (2.3), we set

and obtain

$$
\phi \phi^{\prime 2}=4[(1 / x)-1]
$$

$$
\begin{array}{ll}
\alpha(x)=\frac{2}{3} \phi^{1}=\cos ^{-1} x^{1}-\left(x-x^{2}\right)^{\frac{1}{2}} & 0<x \leq 1 \\
\beta(x)=\frac{2}{3}(-\phi)^{1}=\left(x^{2}-x\right)^{\frac{1}{2}}-\cosh ^{-1} x^{\frac{1}{3}} & 1 \leq x<\infty
\end{array}
$$

with the same conventions about many valued functions as in (8.3). In this case

$$
F(x, \lambda)=F(x)=\frac{1}{2}\{\phi, x\}-\left(1-\alpha^{2}\right) /\left(4 x^{2}\right)
$$

is continuous for $0<x<\infty$ and is $O\left(x^{-2}\right)$ as $x \rightarrow \infty$, and on any interval $0<a<x<\infty$, the conditions of Sec. 2, with $\phi^{\prime}(x)<0$, can easily be verified with $\Lambda(\lambda)$ a suitable constant, and $g(x)=x^{-\frac{1}{2}}|1-x|^{-\frac{1}{2}}$. It follows also that

$$
\int_{x}^{\infty} g(t) d t=O\left(\begin{array}{l}
1 \\
-
\end{array}\right) \quad a<x<\infty .
$$

We now apply the results of Sec. 2 with $m=0, x_{0}=b$. By fixing $n$ and making $x \rightarrow \infty$, we see that

$$
y_{0}(x) / Y_{0}(x) \rightarrow 1 \text {. }
$$

Moreover, in the expression (2.10) for $Y_{0}$, we may use (3.2), and in the resulting asymptotic form,

$$
\beta(x)=x-\log \left(2 x^{\frac{1}{3}}\right)-\frac{1}{2}+O\left(x^{-\frac{1}{3}}\right) \quad x \rightarrow \infty,
$$

and we finally find

$$
y_{0}(x)=\frac{(4 x)^{N} e^{-2 N x+N}}{2(2 \pi)^{\ddagger} N^{1 / 6}}\left[1+O\left(x^{-\frac{1}{2}}\right)\right] \quad x \rightarrow \infty
$$

In particular, $y_{0}(x) \rightarrow 0$ as $x \rightarrow \infty$. It being known that numerical multiples of (8.1) are the only solutions of (8.2) vanishing as $x \rightarrow \infty$, we conclude that (8.1) is a numerical multiple of $y_{0}(x)$. Moreover, from the explicit formula for Laguerre polynomials [7, vol. II, p. 188, Eq. (7)].

$$
\begin{aligned}
x^{(\alpha+1) / 2} e^{-2 N x} L_{n}{ }^{\alpha}(4 N x) & \\
= & \frac{(-4 N)^{n}}{n !} x^{N} e^{-2 N x}\left[1+O\left(x^{-1}\right)\right] \quad x \rightarrow \infty
\end{aligned}
$$

and from a comparison of (8.12) and (8.13), $L_{n}{ }^{\alpha}(4 N x)$ can be expressed in terms of $y_{0}(x)$. Lastly, we use (2.14) and (8.11) to obtain

$$
\begin{aligned}
L_{n}^{\alpha}(4 N x)= & \frac{(-1)^{n}}{n !}\left(\frac{2 \pi}{-\phi^{\prime}}\right)^{\frac{1}{2}} 2^{-\alpha} N^{n+1 / 6} x^{-(\alpha+1) / 2} \\
& \times e^{2 N x-N} A i\left(-N^{3} \phi\right)\left[1+O\left(\frac{1}{N x}\right)\right]
\end{aligned}
$$

valid, with $N=n+(\alpha+1) / 2$ and $\phi$ given by (8.10), for $n=0,1,2, \cdots$ and $0<a<x<\infty$, except that the error term must be modified in the vicinity of zeros of $A i$ which occur when $0<x<1$.

Since we may choose $0<a<b<1$, the regions of validity of the two asymptotic representations (8.9) and (8.14) between them cover the entire real axis. Moreover, these regions overlap. In the common part, $a<x<b$, both forms are valid, the known asymptotic forms may be used for the Bessel function in (8.9) and the Airy function in (8.14), and the asymptotic equivalence of (8.9) and (8.14) for $a<x<b$ follows from the relation $\alpha+\psi^{\frac{1}{2}}=\pi / 2$ which is an immediate consequence of (8.3) and (8.10).

Simpler asymptotic forms may be derived from (8.9) and (8.14). If one is satisfied with an error term $O\left(N^{-1}\right)$, the gamma function and the factorials may be replaced by their approximations obtained from Stirling's formula. In another direction, restriction of $x$ to narrower intervals will make it possible to use approximations for the Bessel functions in (8.9) or the Airy function in (8.14), and will result in asymptotic representations 
involving elementary functions only. Some of the resulting formulas are noted in [6]. Compared with the host of simpler representations, the results developed here have the by no means inconsiderable advantage that two formulas suffice to cover all cases, and that they describe the behaviour of $L_{n}{ }^{\alpha}(x)$ both for unrestricted $n$ as $x \rightarrow 0$ or $x \rightarrow \infty$, and for unrestricted $x$ as $n \rightarrow \infty$.

\section{BIBLIOGRAPHY}

1. H. F. Bohnenblust et al., "Asymptotic solutions of differential equations with turning points, review of the literature," Tech. Rept. 1, Contract Nonr-220(11), Department of Mathematics, California Institute of Technology, 1953.

2. T. M. Cherry, "Uniform asymptotic formulas for functions with transition points," Trans. Am. Math. Soc. 68, 224-257 (1950).

3. A. Erdélyi, "Asymptotic expansions," Tech. Rept. 3, Contract Nonr-220(11), Department of Mathematics, California Institute of Technology, 108 (1955), Reprint, Dover (1956).

4. A. Erdélyi, "Differential equations with transition points. I. The first approximation," Tech. Rept. 6, Contract Nonr220(11), Department of Mathematics, California Institute of Technology, 22 (1955).

5. A. Erdélyi, "Asymptotic solutions of differential equations with transition points," Proc. Intern. Congr. Mathematicians (1954), 3, 92-101 (1956).

6. A. Erdélyi, "Asymptotic forms for Laguerre polynomials," Golden Jubilee Volume of the Indian Mathematical Society.

7. A. Erdélyi et al., Higher Transcendental Functions (McGrawHill Book Company, Inc., 1953-55), 3 Vols.

8. A. Erdélyi, M. Kennedy, and J. L. McGregor, "Parabolic cylinder functions of large order," J. Rat. Mech. and Analysis 3, 459-485 (1954).

9. A. Erdelyi and C. A. Swanson, "Asymptotic forms of Whittaker's confluent hypergeometric functions," Memoirs of the Am. Math. Soc. No. 25 (1957).
10. H. Jeffreys and B. Swirles Jeffreys, Methods of mathematical physics (Cambridge University Press, New York, 1956), 3d ed.

11. N. D. Kazarinoff, "Asymptotic theory of second order differential equations with two simple turning points," Arch. Rat. Mech. Anal. 2, 129-150 (1958).

12. R. E. Langer, "The asymptotic solutions of ordinary linear differential equations of the second order with special reference to the Stokes' phenomenon," Bull. Am. Math. Soc. 40, 545582 (1934).

13. R. E. Langer, "The asymptotic solutions of ordinary linear differential equations of the second order, with special reference to turning points," Trans. Am. Math. Soc. 67, 461-490 (1949).

14. R. E. Langer, "Asymptotic theories for linear ordinary differential equations depending upon a parameter," J. Soc. Indust. Appl. Math. 7, 298-305 (1959).

15. J. C. P. Miller, "The Airy integral" in B.A.A.S. Math. Tables, Part-Volume B, (Cambridge University Press, New York, 1946).

16. F. W. J. Olver, "The asymptotic solutions of linear differential equations of the second order for large values of a parameter and the asymptotic expansion of Bessel functions of large order," Phil. Trans. Roy. Soc. (London) A247, 307-368 (1954).

17. F. W. J. Olver, "The asymptotic solution of linear differential equations of the second order in a domain containing one transition point," Phil. Trans. Roy. Soc. (London) A249, 65-97 (1956).

18. F. W. J. Olver, "Uniform asymptotic expansions of solutions of linear second-order differential equations for large values of a parameter," Phil. Trans. Roy. Soc. (London) A250, 479-517 (1958).

19. H. Skovgaard, "Asymptotic forms of Hermite polynomials," Technical Report 18, Contract Nonr-220(11), Department of Mathematics, California Institute of Technology, 20 (1959).

20. C. A. Swanson, "Differential equations with singular points," Tech. Rept. 16, Contract Nonr-220(11), Department of Mathematics, California Institute of Technology, 24 (1956).

21. G. Szegö, Orthogonal Polynomials (American Mathematical Soc. Colloquium Publications, 1959), Vol. 23, 2d ed.

22. F. G. Tricomi, "Sul comportamento asintotico dei polinomi di Laguerre," Ann. Mat. Pura Appl. (4) 28, 263-289 (1949). 